\title{
“New-Look” Opioids: Biased Ligands
}

\author{
Joseph V. Pergolizzi'1 ${ }^{1}$ Michael H. Ossipov², Robert Taylor ${ }^{1}$, Robert B. Raffa ${ }^{3,4}$ \\ ${ }^{1}$ NEMA Research Inc., Naples, FL, USA \\ ${ }^{2}$ College of Medicine, University of Arizona, Tucson, AZ, USA \\ ${ }^{3}$ College of Pharmacy, University of Arizona, Tucson, AZ, USA \\ ${ }^{4}$ Temple University, School of Pharmacy, Philadelphia, PA, USA \\ Email: robert.raffa@temple.edu
}

How to cite this paper: Pergolizzi, J.V., Ossipov, M.H., Taylor, R. and Raffa, R.B. (2018) "New-Look" Opioids: Biased Ligands. Pharmacology \& Pharmacy, 9, 242-249.

https://doi.org/10.4236/pp.2018.97018

Received: June 12, 2018

Accepted: July 15, 2018

Published: July 18, 2018

Copyright $\odot 2018$ by authors and Scientific Research Publishing Inc.

This work is licensed under the Creative

Commons Attribution International

License (CC BY 4.0).

http://creativecommons.org/licenses/by/4.0/

\begin{abstract}
Between the illicit use of opioids and attendant overdoses, and accidental overdoses with prescribed drugs, overuse of opioids has become a serious problem. At the same time, finding that fine balance between minimizing the risk of opioid misuse and abuse while at the same time providing access to treatment for patients who need pain control presents an ongoing challenge. Efforts to discover and develop better agents have led to what we term "new-look" opioids. We summarize here one such approach-known as biased ligands. By targeting a subset of GPCR signal transduction, this approach attempts to increase the separation between therapeutic and adverse effects.
\end{abstract}

\section{Keywords}

Pain, Analgesic Drugs, Opioids, G Protein-Coupled Receptors, Biased Ligands

\section{Introduction}

\subsection{Global Burden of Pain and the Need for Pain Medications}

Chronic (or persistent) pain-generally loosely defined as pain persisting for more than three months-remains among the leading causes of disability, and years lived with disability (YLD). According to The Global Burden of Diseases, Injuries, and Risk Factors Study 2016, of the 59 conditions with a global prevalence exceeding $1 \%$, seven of these are primary pain conditions: tension-type headache, migraine, lower back pain, neck pain, "other musculoskeletal conditions", osteoarthritis, and urolithiasis (kidney stones) [1] [2]. Taking into account both severity and prevalence, the YLDs provide an overall picture of disease burden. Globally, low back pain and migraine were the two leading causes of YLDs, responsible for 57.6 million and 45.1 million total YLDs, respectively 
[1]. Chronic neck pain and "other musculoskeletal disorders" were each responsible for 29 million YLDs and osteoarthritis for 16 million YLDs [1]. The burden of chronic pain is actually even greater than this, because these numbers do not reflect conditions such as cancers, sickle cell anemia, diabetic neuropathy, neuropathic conditions, and many others, where pain is but one of many symptoms [2]. Thus, chronic pain is a global problem that severely impacts the quality of life of those suffering from it, and it exacts a significant socioeconomic toll. Lost productivity due to pain is measured in the billions of dollars.

\subsection{Increase in Opioid Use for Non-Cancer Chronic Pain and Potential Consequences}

Prior to the late 1970's and 1980's the management of non-cancer chronic pain, and even of some cancer pains, was almost absent. Opioids were generally only used for postoperative pain, severe acute pain, and end-of-life care. And even as late as 2008, The World Cancer Declaration felt the need to include a target to make effective pain control more accessible [3]. The use of opioids for chronic non-cancer pain, although still considered to have unproven efficacy, consistently increased over the past two decades [4].

Although several randomized clinical trials have supported short-term use of opioids for chronic non-cancer pain, including neuropathic pain, long-term use of opioids has not been as rigorously studied [5]. An observational, descriptive study of 84 patients with intractable, severe, daily non-cancer chronic pain that was conducted over one year found that these patients reported at least $50 \%$ or greater pain relief and a moderate improvement in disability [5]. In that select population, problematic opioid use, tolerance and serious adverse effects were not considered to be "major issues." Thus, opioids used in accordance with established guidelines, carefully monitored, and used in a multidisciplinary chronic pain practice can be a safe and effective approach to treating chronic pain. This is an important caveat. Realistically, though, most opioid prescriptions are not written by chronic pain specialists, but by primary care physicians who may have incomplete understanding of these drugs, and an overconfidence in their relative safety [4] [6]. Indeed, a perception arose that opioids are virtually risk-free if taken as directed by a physician, which is not true. This is reflected in a comprehensive review that found that $20 \%$ of opioid-related deaths occurred in patients on low-dose opioid therapy of $100 \mathrm{mg}$ of morphine equivalent dose or less per day and $40 \%$ in those receiving morphine of over $100 \mathrm{mg}$ per day [7]. The remaining $40 \%$ of deaths occurred in individuals abusing the drugs obtained through multiple prescriptions, doctor shopping, and drug diversion. In the same study, it was reported that only $16 \%$ of users of non-therapeutic opioids reported that they obtained the drug by prescription from a doctor, while more than half of individuals reported obtaining the drug from a friend or relative.

Between 1999 and 2010, the number of prescriptions written for opioids in the United States quadrupled, largely due to prescribing of opioids for non-cancer chronic pain [4]. The increase in opioid prescriptions has unfortunately been 
associated with a contemporaneous increase in opioid overdoses, emergency room visits, and deaths, as well as an increase in admissions for treatment of opioid use disorders. [4] [6]. The rise in opioid prescriptions reached a peak in 2012, and has since declined, but the rise in opiate overdoses has continued. This is partly due to the increased use of illicit heroin and fentanyl (and congeners). The role of increased availability of prescription drugs to the current opioid epidemic has not been rigorously examined, and the causes are multiple and complex. Use or misuse/abuse of opioid analgesics was responsible for more deaths than suicide and motor vehicle accidents together, or from cocaine and heroin combined [7]. This is not a new dilemma. We are reminded that the invention of the hypodermic needle in the mid 1800's, permitting morphine injection, was a boon to soldiers suffering from horrific wounds during the Civil War. But this blessing was followed by the curse of "Soldier's Disease"-Civil War veterans who had become addicted to morphine.

\subsection{Approaches to Better Opioids}

The rather dramatic rise in opioid use disorders and opioid-related deaths, regardless of cause, has spurred several approaches to develop or reconsider novel, safer opioid drugs. Approaches include pharmacodynamics, influencing the action of opioids at the opioid receptor, pharmacokinetic, such as limiting the ability of the drug to cross the blood-brain barrier, combination therapies to limit the exposure to opioids, and preparation of abuse-resistant formulations. We call these "new-look" opioids, and present a summary of one of these approaches here. We present the conceptual framework for this approach rather than a list of the compounds in development. We discuss one drug as a representative example.

\section{The Opioid Receptor and Opioid Analgesic Activity}

The early to mid 1970's was an exciting time in pain research. Within a short period, it was discovered that activation of specific brain sites could produce powerful antinociception in animal models and analgesia in patients with intractable pain. The contemporaneous discovery of a receptor specific for opioids suggested not only that drugs such as morphine and codeine could activate specific pain-modulating pathways in the central and peripheral nervous systems, but it also suggested that there were endogenous substances that exist to activate these receptors. The discovery of the enkephalins, endorphin, and dynorphin confirmed the existence of endogenous substances that exist to activate the opioid receptor and produce analgesia. Further studies led to the identification of three opioid receptor subtypes, which were named based on putative ligands that preferentially bind to them, or the tissue system where they were characterized. Thus, we have the $\mu$ (morphine), $\kappa$ (cyclazocine), and $\delta$ (mouse vas deferens) opioid receptors. More recently, the nociception (ORL1) and the $\zeta$ opiate receptors were added to the family. It was hoped that as our understanding of these 
receptors and their functions increased, the development of safer, highly effective analgesics with little to no abuse liability would follow. There were several attempts at developing analgesics acting at the $\kappa$ and $\delta$ opiate receptors, but they were abandoned because of adverse effects such as dysphoria $(\kappa)$ and convulsions $(\delta)$. However, recent discoveries relating to the intracellular mechanisms associated with the $\mu$-opioid receptor may led us closer to this holy grail of pain management.

\subsection{Molecular Biology and Signaling Pathways of the $\mu$-0pioid Receptor}

The functioning of the $\mu$-opioid receptor, a member of the $\mathrm{G}$ protein-coupled receptor (GPCR) superfamily, has received considerable attention [8] [9] [10] [11]. These receptors consist of seven-transmembrane domains, an extracellular $N$-terminal, and an intracellular $C$-terminal. The extracellular terminal and loops of the transmembrane segments form the receptor binding and recognition sites. The intracellular portions of the receptor bind to the inhibitory $G$ protein, consisting of $G_{a i / o}, G_{\beta}$, and $G_{\gamma}$ subunits. Binding of an opioid agonist to the receptor releases the $G$ protein complex, and $G_{\text {ai/o }}$ can activate inward rectifying $\mathrm{K}^{+}$channels, while the $\mathrm{G}_{\beta / \gamma}$ subunits inhibit $\mathrm{Ca}^{2+}$ channels, leading to hyperpolarization and decreased neuronal excitability (Figure 1). In addition, the $\mathrm{G}_{\mathrm{\alpha} i / \mathrm{o}}$ subunit inhibits adenylate cyclase, decreasing cyclic adenosine monophosphate production (cAMP), and thus inhibiting downstream signaling cascades dependent on cAMP. The attachment of the agonist to the receptor also allows phosphorylation of the intracellular segments of the receptor by protein kinase A or C (PKA or PKC), or by a corresponding GPCR kinase (GRK). The phosphorylated receptor recruits $\beta$-arrestins, so named since they arrest the activity of the receptor. Once bound to the receptor, the $\beta$-arrestins complex with components of the receptor endocytotic process, resulting in internalization and recycling of the receptor.

They inhibit the influx of presynaptic $\mathrm{Ca}^{2+}$, thereby decreasing neurotransmitter release and firing of postsynaptic neurons, and they hyperpolarize postsynaptic neurons, thereby rendering them less likely to fire in response to excitatory input from presynaptic neurons.

The binding of an agonist to $\mu$-opioid receptors in presynaptic neurons results in inhibition of $\mathrm{Ca}^{2+}$ influx, and thus less neurotransmitter release, in painsensing/transmitting pathways. The binding of an agonist to $\mu$-opioid receptors in postsynaptic neurons results in enhanced $\mathrm{K}^{+}$efflux, hyperpolarization, and less responsiveness in pain-sensing/transmitting pathways. Illustration is from Wikimedia Commons.

\section{2. "Biased" Signaling}

Emerging evidence indicates that the $\beta$-arrestins can also mediate alternate signaling pathways, independent of $G$ proteins, involving mitogen-activated 


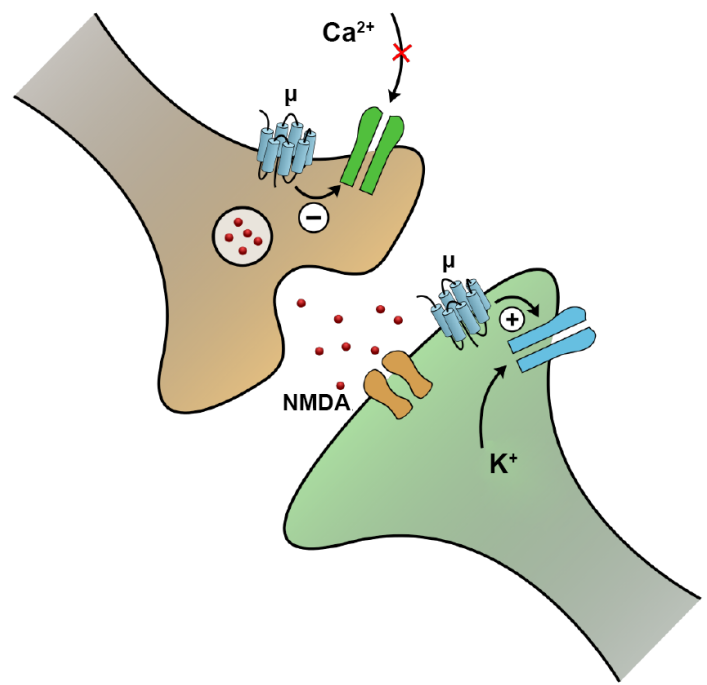

Figure 1. Opioids produce presynaptic and postsynaptic effects that decrease response to excitatory (pain) input. They inhibit presynaptic $\mathrm{Ca}^{2+}$ influx, decreasing neurotransmitter release, and hyperpolarize postsynaptic neurons, rendering them less likely to fire in response to excitatory input. The binding of agonist to $\mu$-opioid receptors in presynaptic neurons inhibits $\mathrm{Ca}^{2+}$ influx and thus neurotransmitter release. The binding of agonist to $\mu$-opioid receptors in postsynaptic neurons enhances $\mathrm{K}^{+}$ efflux and hyperpolarizes postsynaptic neurons. Illustration from Wikimedia Commons

protein kinases (MAPK), such as extracellular signal-regulated kinases 1 and 2 (ERK1/2), JNK1-3, and p38 ( $\alpha, \beta, \gamma, \delta)$ stress kinase [11] [12] [13] [14]. The MAPKs are able to transmit a diverse array of intra- and extra-cellular signals and can influence gene transcription. The discovery that genetically altered mice that do not express $\beta$-arrestin still respond to opioids with antinociception-but with less respiratory depression-led to the growing viewpoint that activating the $\mu$-opioid receptor without engaging $\beta$-arrestin regulation may be key to the development of safer opioid analgesics [12] (Figure 2). Morphine, acting through both pathways is considered to be "unbiased" to the pathways. Fentanyl, which is associated with greater respiratory depression than morphine, shows a "bias" towards the $\beta$-arrestin pathway. Based on these observations, Schmid and colleagues developed a series of compounds derived from a piperidine core structure [12]. Using differences in relative agonist activity between two assays, a bias factor for activation of the G protein and $\beta$-arrestin pathways was determined for each of the compounds. These were then correlated with the therapeutic window derived from nociceptive assays and measures of respiratory depression in mice for each of the compounds. A strong correlation was found between the therapeutic window and signaling bias. Compounds such as fentanyl, with a greater bias towards $\beta$-arrestin, are more likely to produce respiratory depression even at low doses, whereas those with $G$ protein signaling bias 


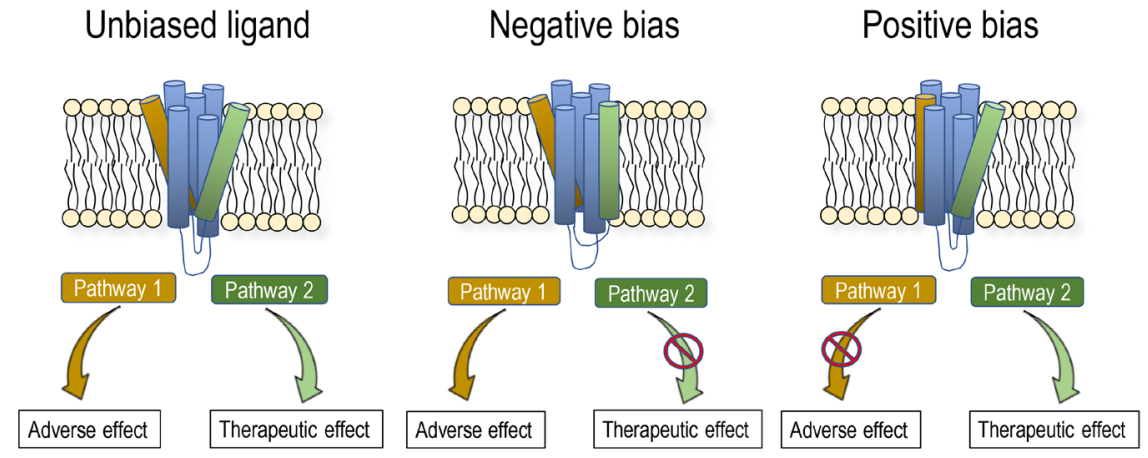

Figure 2. Biased ligand concept. Selectively targeting the G protein-mediated pathway, and avoiding the $\beta$-arrestin pathway, increases the separation between the desired therapeutic and the unwanted adverse effects of a drug. From http://www.trevena.com/technology.php.

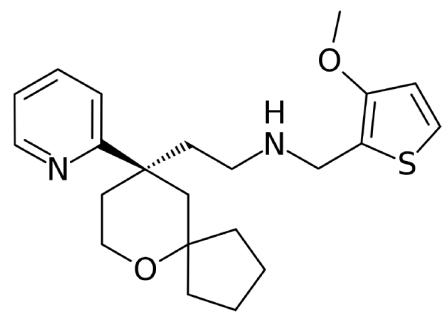

Figure 3. Chemical structure of the biased ligand oliceridine (TRV130).

have broader therapeutic windows. It is hoped that studies such as this one helps further the development of safer alternatives to current opioid therapeutics.

\section{Current Status}

Oliceridine (TRV130) (Figure 3) was developed by Trevena Inc. as a G protein based ligand at the $\mu$-opioid receptor [12]. In preclinical studies, it produced less respiratory depression and gastrointestinal dysfunction than morphine at equianalgesic doses. In a phase II trial in patients following abdominoplasty, intravenous oliceridine produced a lower prevalence of nausea, vomiting, and depressed respiratory function compared to morphine [15]. Oliceridine received Breakthrough Therapy Designation from the U.S. Food and Drug Administration (FDA), and the New Drug Application for the i.v. formulation is currently under review by the FDA. These developments suggest that biased ligands may provide an avenue to a safer, potent, and efficacious opioid analgesic.

\section{Perspective and Conclusions}

The medical and broader communities face a dual epidemic: under-treatment of pain and over-use of drugs used to treat pain. Chronic pain severely impacts the quality of life of those suffering from it, and it exacts a significant toll on the patient, the healthcare professions, and the community at large. Opioid analgesics mimic the body's own pain attenuation physiology, and can be effective pain re- 
lievers. But the traditional opioids suffer from a questionable safety profile. Researchers are actively searching for "new-look"-safer-opioid analgesics. Biased-ligand is one such promising approach. The regulatory fate and clinical proof-of-principle are eagerly awaited.

\section{References}

[1] Vos, T., Abajobir, A.A., Abate, K.H., Abbafati, C., Abbas, K.M., Abd-Allah, F., et al. (2017) Global, Regional, and National Incidence, Prevalence, and Years Lived with Disability for 328 Diseases and Injuries for 195 Countries, 1990-2016: A Systematic Analysis for the Global Burden of Disease Study 2016. The Lancet, 390, 1211-1259 https://doi.org/10.1016/S0140-6736(17)32154-2

[2] Rice, A.S., Smith, B.H. and Blyth, FM. (2016) Pain and the Global Burden of Disease. PAIN, 157, 791-796. https://doi.org/10.1097/j.pain.0000000000000454

[3] National Collaborating Centre for Cancer (2012) Opioids in Palliative Care: Safe and Effective Prescribing of Strong Opioids for Pain in Palliative Care of Adults. NHS NICE Clinical Guideline. https://www.ncbi.nlm.nih.gov/pubmed/23285502

[4] Tolba, R., Meselhy, E. and Guerra, C.E. (2018) The Opioid Epidemic and Pain Medicine Specialists: Where to Begin and What Is Next? The Ochsner Journal, 18, 20-22.

[5] Watson, C.P., Watt-Watson, J. and Chipman, M. (2010) The Long-Term Safety and Efficacy of Opioids: A Survey of 84 Selected Patients with Intractable Chronic Noncancer Pain. Pain Research and Management, 15,213-217.

https://doi.org/10.1155/2010/867201

[6] Brown, R.E. and Sloan, P.A. (2017) The Opioid Crisis in the United States: Chronic Pain Physicians Are the Answer, Not the Cause. Anesthesia \& Analgesia, 125, 1432-1434. https://doi.org/10.1213/ANE.0000000000002417

[7] Manchikanti, L., Helm 2nd, S., Fellows, B., Janata, J.W., Pampati, V., Grider, J.S., et al. (2012) Opioid Epidemic in the United States. Pain Physician, 15, ES9-ES38.

[8] Raehal, K.M., Schmid, C.L., Groer, C.E. and Bohn, L.M. (2011) Functional Selectivity at the $\mu$-Opioid Receptor: Implications for Understanding Opioid Analgesia and Tolerance. Pharmacological Reviews, 63, 1001-1019.

[9] Childers, S.R. (1991) Opioid Receptor-Coupled Second Messenger Systems. Life Sciences, 48, 1991-2003.

[10] Knapp, R.J., Malatynska, E., Collins, N., Fang, L., Wang, J.Y., Hruby, V.J., et al. (1995) Molecular Biology and Pharmacology of Cloned Opioid Receptors. FASEB Journal, 9, 516-525. https://doi.org/10.1096/fasebj.9.7.7737460

[11] Al-Hasani, R. and Bruchas, M.R. (2011) Molecular Mechanisms of Opioid Receptor-Dependent Signaling and Behavior. Anesthesiology, 115, 1363-1381.

[12] Schmid, C.L., Kennedy, N.M., Ross, N.C., Lovell, K.M., Yue, Z., Morgenweck, J., et al. (2017) Bias Factor and Therapeutic Window Correlate to Predict Safer Opioid Analgesics. Cell, 171, 1165-1175. e13. https://doi.org/10.1016/j.cell.2017.10.035

[13] DeWire, S.M., Yamashita, D.S., Rominger, D.H., Liu, G., Cowan, C.L., Graczyk, T.M., et al. (2013) A G Protein-Biased Ligand at the $\mu$-Opioid Receptor Is Potently Analgesic with Reduced Gastrointestinal and Respiratory Dysfunction Compared with Morphine. Journal of Pharmacology and Experimental Therapeutics, 344, 708-717.

[14] Rankovic, Z., Brust, T.F. and Bohn, L.M. (2016) Biased Agonism: An Emerging Pa- 
radigm in GPCR Drug Discovery. Bioorganic \& Medicinal Chemistry Letters, 26, 241-250. https://doi.org/10.1016/j.bmcl.2015.12.024

[15] Singla, N., Minkowitz, H.S., Soergel, D.G., Burt, D.A., Subach, R.A., Salamea, M.Y. et al. (2017) A Randomized, Phase IIb Study Investigating Oliceridine (TRV130), a Novel Micro-Receptor G-Protein Pathway Selective ( $\mu$-GPS) Modulator, for the Management of Moderate to Severe Acute Pain Following Abdominoplasty. Journal of Pain Research, 10, 2413-2424. 